\title{
Influence of Carbon Nanotube Surface Treatment on Resistivity and Low-Frequency Noise Characteristics of Epoxy-Based Composites
}

\author{
Marina Tretjak, ${ }^{1}$ Sandra Pralgauskaite, ${ }^{1}$ Jan Macutkevic $\mathbb{D D}^{1},{ }^{1}$ Jonas Matukas, ${ }^{1}$ Juras Banys, ${ }^{1}$ \\ Polina Kuzhir, ${ }^{2,3}$ Evgeni Ivanov, ${ }^{4}$ Rumiana Kotsilkova ${ }^{4}$ \\ ${ }^{1}$ Vilnius University, Vilnius, LT-10257, Lithuania \\ ${ }^{2}$ Institute for Nuclear Problems of Belarusian State University, Minsk 220030, Belarus \\ ${ }^{3}$ Tomsk State University, Tomsk 634050, Russia \\ ${ }^{4}$ OLEM, Institute of Mechanics, Bulgarian Academy of Sciences, Sofia, Bulgaria
}

Resistivity and low-frequency $(10 \mathrm{~Hz}-20 \mathrm{kHz})$ noise characteristics of composite materials with multi-walled carbon nanotubes (MWCNTs) of different surface treatment, that is, MWCNTs covered with bisphenol-A based liquid epoxy resin (epoxy-grafted) and polyethylene polyamine (amino-grafted), have been carried out over the temperature range from 73 to $380 \mathrm{~K}$. The resistivity of the investigated materials decreases with temperature increase up to $250 \mathrm{~K}$; at higher temperatures polymer matrix expansion leads to the resistivity increase and above $340 \mathrm{~K}$ the conductivity in the matrix becomes significant. Lowfrequency noise spectra of the investigated materials are $1 / f^{\alpha}$-type and noise spectral density is proportional to the squared voltage. The observed fluctuations in the investigated materials are resistance fluctuations. The conduction in the investigated composites is caused by tunneling inside and between MWCNTs controlled by charge carrier capture and release processes in localized states. MWCNT's surface treatment by polyethylene polyamine leads to the larger density of surface states what causes lower resistivity and more intensive lowfrequency fluctuations. POLYM. COMPOS., 00:000-000, 2018. (C) 2018 Society of Plastics Engineers

\section{INTRODUCTION}

Composites filled with carbon nanoparticles attract great attention as novel material for various multifunctional nanodevices: bioelectronic devices, nanosized

Correspondence to: J. Macutkevic; e-mail: jan.macutkevic@gmail.com Contract grant sponsor: H2020 RISE 734164 Graphene 3D project and Lithuanian-Belorussian collaboration program project; contract grant number: S-LB-17-2/LSS-120000-143.

DOI 10.1002/pc.24775

Published online in Wiley Online Library (wileyonlinelibrary.com).

(C) 2018 Society of Plastics Engineers field-effect transistor (FETs), sensors, and so forth [1-7]. Devices fabricated using materials with carbon nanoparticles are distinguished by desired electrical, chemical and thermal characteristics, large current carrying capacity, vapor and ultraviolet (UV) absorption, fast response, high mechanical stability and flame resistance. Carbon nanotube (CNT)-based FETs are proposed as alternative for Si-based Complementary metal oxide semiconductor FETs since CNTs help to cope with a short channel effects in FETs when transistor size is reduced [8-10]. Using CNTs (that are only 1-nm thick) enables to reduce the device gate length to 10 nanometers [9].

Multi-walled CNT (MWCNT) can be treated as conductive particles (that make up a percolation network) in polymer matrix. Electromagnetic properties of CNT composites are greatly dependent on nanotubes geometrical parameters, aspect ratio, number of walls, functionalization type, density, orientation, and distribution in the matrix [11, 12]. Depending on all the mentions factors and many others both metallic and non-metallic behavior and conductivity variation with temperature can be observed [13]. Much depends on the properties of the CNTs themselves. Grafting with the conductive polymers onto CNTs is effective method for their surface modification and obtaining of desired qualities [14]. It is shown that the chemical functionalization of nanotube surface improves the compatibility of CNTs with the polymer matrix and has positive influence on rheological, electrical, thermal, mechanical, optical and other properties of polymer composites [15-17]. CNT's surface modification leads to the changes of the dielectric behavior and the electrical conductivity $[12,15,17,18]$. 
Investigation of carrier transport mechanisms in such disordered materials as CNT composites is important for many of their applications. Two microscopic carrier transport mechanisms can be distinguished in the percolative network: the transport along nanotubes themselves and the transport between nanotubes. There is a number of papers dealing with modeling of the electrical conductivity in CNT composites [15-20]. However, experimental studies of carrier transport in these materials are rare.

The low-frequency noise spectroscopy is a very sensitive experimental tool that gives information on physical processes and enables clearing-up the conduction mechanisms in composite materials [21-24]. It gives a valuable information on the charge carrier transport and conduction mechanisms in new materials [17, 19, 25-27]. On the other hand, $1 / f$ noise is an important investigation in assessing the potential of CNTs for electronic and sensor applications. In the most cases, the low-frequency noise is a limiting factor for signal detection. It is reported that in the materials with CNTs mainly $1 / f$-type fluctuations are observed [17, 18, 25]. The noise in such composites is caused by impurities in nanotubes or defects in their structure. Both low-frequency noise characteristics and conductivity depend on the nanoparticles' dimensions, their density and distribution in the matrix [1, 2, 26, 28]. It is shown that the $1 / f$ noise is rather high in films with small CNTs concentration, whereas the noise level decreases when the CNTs density increases [17]. The large value of $1 / f$ noise observed in CNT composites also can be attributed to the fact that the electrical current in nanotubes is transmitted through the surface and it is easily perturbed by the local charge fluctuations $[25,26]$. The surface area of CNTs is extremely large as almost all atoms that constitute CNT are surface atoms $[1,26]$. That is the contribution of the fluctuations in the material in which CNTs are embedded to the low-frequency noise should be taken into account [27, 28].

In this article, we present comprehensive investigation of low-frequency noise and resistivity characteristics of composite materials loaded with MWCNT with different surface treatment. The aim of the investigation was to determine the charge carrier transport mechanisms in the investigated composite materials and to clarify the influence of the MWCNT surface treatment to the material conduction and noise properties.

\section{EXPERIMENTAL}

\section{Materials}

Commercially available (Nanocyl) MWCNT synthesized by CVD method having external diameter $\sim 30 \mathrm{~nm}$ and approximate length 10-20 $\mu \mathrm{m}$ were used for fabrication of the investigated composite materials [15]. The Dow Chemical (D.E.R. 321) bisphenol-A based liquid epoxy resin was chosen as a polymer matrix. The polyethylene amine (PEA) provided by the Bakelite Co was used as a hardener. Two kinds of MWCNT/epoxy composites were investigated in this study: "epoxy-grafted" and "amino-grafted". A sonochemical method was employed for in situ surface modification of CNTs, in both epoxy resin and polyethylene polyamine hardener, using a methodology described in our previous study [15]. Solid composites were prepared after curing at molar ratio D.E.R:PEA (70:30). CNTs dispersion in different solution/or chemicals leads to the different properties of CNT surface, that have the impact on electrical, thermal, and other properties of polymer composites [15-17]. The percolation threshold of the obtained materials is in the range of 0.03-0.05 wt\% for epoxy-grafted, and 0.05-0.08 wt $\%$ for amino-grafted samples [15]. For investigations were selected composites with concentrations: $0.3 \mathrm{wt} \%$ (in the paper referred as "E0.3" for "epoxy-grafted" sample and "A0.3" for "amino-grafted" one). Therefore, materials with $0.3 \mathrm{wt} \%$ of MWCNTs are far above the percolation threshold, while materials with 0.08 wt $\%$ conductive inclusions are just over it. Rectangular samples with typical thickness $1.25 \mathrm{~mm}$ and area $15 \mathrm{~mm}^{2}$ were investigated. Silver contacts were used for the measurements.

\section{Measurement Technique}

The low-frequency fluctuations were investigated at 10 $\mathrm{Hz}$ to $20 \mathrm{kHz}$ frequencies at temperatures ranging from $73 \mathrm{~K}$ to $380 \mathrm{~K}$. The voltage noise was measured under constant current operation: the load resistance, $R_{\text {load, }}$, was at least 30 times larger comparing to the resistance of the sample under test. The measured noise signal was processed by low-noise amplifier, filter system, and analog digital converter (National Instrument PCI 6115 board) [29]; and then fast Fourier transform was performed. The voltage noise spectral density, $S_{U}$, was evaluated by comparing to the thermal noise of the standard resistor, which was at room temperature, and was used also as the load resistor:

$$
S_{U}=\frac{\overline{U^{2}}-\overline{U_{\mathrm{s}}^{2}}}{\overline{U_{\mathrm{st}}^{2}}-\overline{U_{\mathrm{s}}^{2}}} 4 k T_{0} R_{\text {load }}
$$

where $\overline{U^{2}}, \overline{U_{s}^{2}}$, and $\overline{U_{s t}^{2}}$ are, respectively, sample, measuring system, and standard (load) resistor thermal noise variances in the narrow frequency band $\Delta f ; T_{0}$ is the absolute temperature of the standard resistor, and $k$ is the Boltzmann constant. The noise spectral density of a resistor, according the Nyquist's theorem, depends entirely on its resistance and temperature and can be used as a reference.

The noise measurements were performed in a specially shielded room (Faraday cage) in order to avoid interferences from electrical network and communication systems.

The fracture morphology of the cured composites was studied by scanning electron microscopy (SEM FEI 

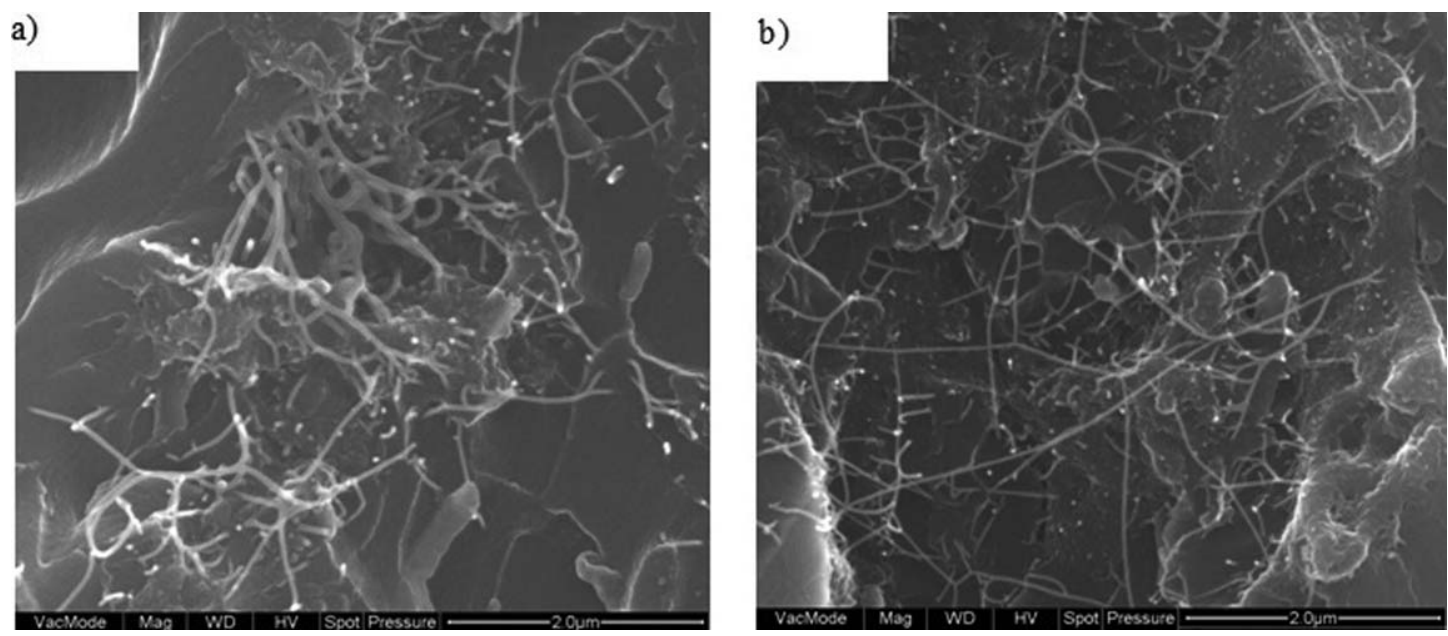

FIG. 1. SEM micrograph of 0.3\% MWCNT/epoxy composite (a) amino-grafted; (b) epoxy-grafted, at magnification $50000 \times$.

Quanta 600F, at CfAM University of Reading, UK) in micro and nanoscale at various magnifications. Samples were cut in liquid nitrogen and coated with vacuum evaporated chromium.

\section{RESULTS AND DISCUSSION}

\section{Resistivity and Structure of Composites}

Figure 1 presented the SEM micrographs of (a) aminografted and (b) epoxy-grafted MWCNT/epoxy composites containing $0.3 \mathrm{wt} \%$ filler contents. As seen, the aminografted MWCNTs attracted a thicker polymer layer on their surfaces compared with the epoxy-grafted nanotubes, but the latest were better dispersed in the epoxy resin matrix.

The resistivity of investigated materials is a constant in particular voltage range: (10-100) $\mathrm{mV}$ at room

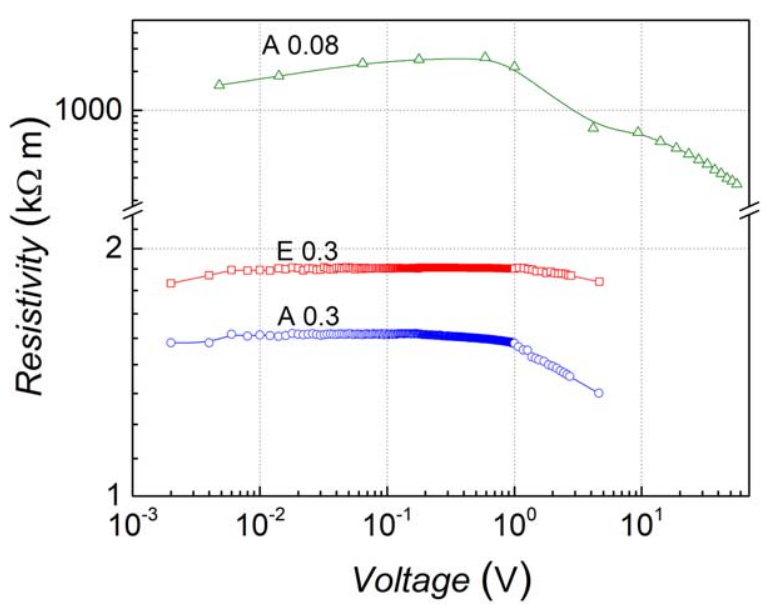

FIG. 2. Dependences of the resistivity for MWCNT composites on voltage at room temperature. [Color figure can be viewed at wileyonlinelibrary.com] temperature (Fig. 2). Thus, the current-voltage characteristic is linear at this voltage range. Above approximately $1 \mathrm{~V}$, the resistance of the material starts to decrease and the current-voltage characteristic becomes super-linear. As the composite with MWCNTs is a material with conductive fillers, it is typically described by the Ohmic resistivity at low electrical field and the resistivity decreases at higher voltage $[30,31]$.

The resistivity dependence on the temperature is presented in Fig. 3. The resistivity of the composites E0.3 and A0.3, decreases with temperature increase. For composites A0.08 the decrease of the resistivity is observed below room temperature and above $325 \mathrm{~K}$, while in temperature range 300-325 $\mathrm{K}$ the resistivity increases with temperature. In the temperature range $(80-130) \mathrm{K}$ the resistivity dependence on temperature can be well described by the fluctuation-induced tunneling model [31, 32]:
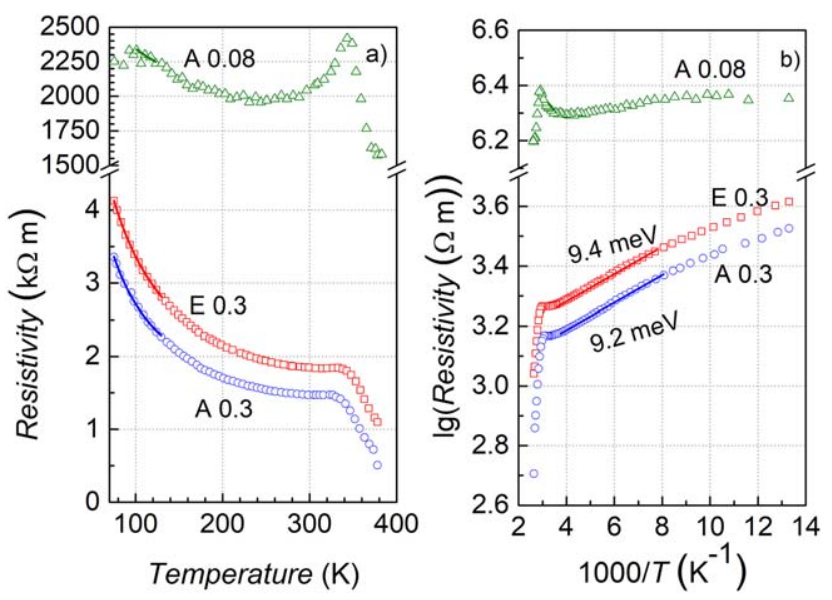

FIG. 3. Dependences of the resistivity for MWCNT composites on direct (a) and inverse (b) temperature at $1 \mathrm{~V}$ voltage (symbols-experimental data, solid lines - aproximations: (a) by Eq. (1), (b) by Arrhenius law). [Color figure can be viewed at wileyonlinelibrary.com] 
TABLE 1. Parameters of the approximations of resistivity versus temperature dependency by Eq. (1) and Arrhenius law.

\begin{tabular}{|c|c|c|c|c|}
\hline \multirow[b]{2}{*}{ Materials } & \multicolumn{2}{|c|}{$\begin{array}{c}80-130 \mathrm{~K} \\
\text { (approximation } \\
\text { by } E q .1 \text { ) }\end{array}$} & \multirow{2}{*}{$\frac{130-250 \mathrm{~K}}{\Delta E, \mathrm{meV}}$} & \multirow{2}{*}{$\begin{array}{c}T_{\mathrm{C}}, \mathrm{K} \\
\text { (evaluated } \\
\text { from Fig. 3) }\end{array}$} \\
\hline & $T_{0}, \mathrm{~K}$ & $T_{1}, \mathrm{~K}$ & & \\
\hline E0.3 & 71 & 207 & 9.4 & 130 \\
\hline $\mathrm{A} 0.3$ & 37 & 132 & 9.2 & 133 \\
\hline A0.08 & 30 & 35 & 3.7 & 125 \\
\hline
\end{tabular}

$$
\rho=\rho_{0} \exp \left(\frac{T_{1}}{T+T_{0}}\right)
$$

where $T$ is the temperature, $T_{0}$ is the characteristic temperature below which the dc conductivity reduces to temperature-independent tunneling, and $T_{1}$ reflects the energy required to move an electron across the insulating gap between conductive aggregates. Therefore, the carrier transport at low temperature occurs due to the electrons tunneling between clusters. The parameters obtained from the fit of the tunneling model (Eq. 2) to the experimental data are presented in Table 1 .

On the other hand the resistivity of composites follows the Arrhenius law at higher temperatures (Fig. 3b) indicating that the conduction mechanism based on the thermal activation over the potential barrier is predominant. Indeed, the conductivity based on the thermal activation over the potential barrier can be described by the following Equation [31, 33]:

$$
\rho=\rho_{0} \exp \left(\frac{T_{c} T_{1}}{T\left(T c+T_{0}\right)}\left(1-\varepsilon_{A}\right)^{2}\right)=\rho_{0} \exp \left(\frac{\Delta E}{T}\right) ;
$$

where $T_{\mathrm{c}}$ is the crossover temperature above which the fluctuation-induced tunneling and the thermal activation contributions are indistinguishable, $\varepsilon_{A}$ is the dimensionless applied electric field, and $\Delta E=\frac{T_{c} T_{1}}{T_{c}+T_{0}}$. For the epoxy with $0.3 \mathrm{wt} \%$ MWCNTs inclusions the value of parameter $\Delta E$ in the temperature range $130-250 \mathrm{~K}$ is $9.2-9.4 \mathrm{meV}$ (Fig. 3b). For A0.08 sample the thermal activation process is masked by the expansion of the polymer matrix, which increases resistivity and is more noticeable in materials with low concentration of conductive inclusions. Indeed, in the temperature range around the room temperature (starting from $\sim 280 \mathrm{~K}$ ) the resistivity of composites with 0.3 wt $\%$ MWCNTs is almost the constant and it increases with temperature (starting from $240 \mathrm{~K}$ ) for materials with $0.08 \mathrm{wt} \%$ inclusions (Fig. 3). As behavior of both types-amino-grafted and epoxy-grafted, CNT based composites is the same in this temperature region, this effect should be attributed to the polymer matrix behavior, but not with MWCNTs. The value of $240 \mathrm{~K}$ is close to the epoxy glass transition temperature [34]. Above this temperature, the resistivity of composites increases due to the rapid expansion of the polymer

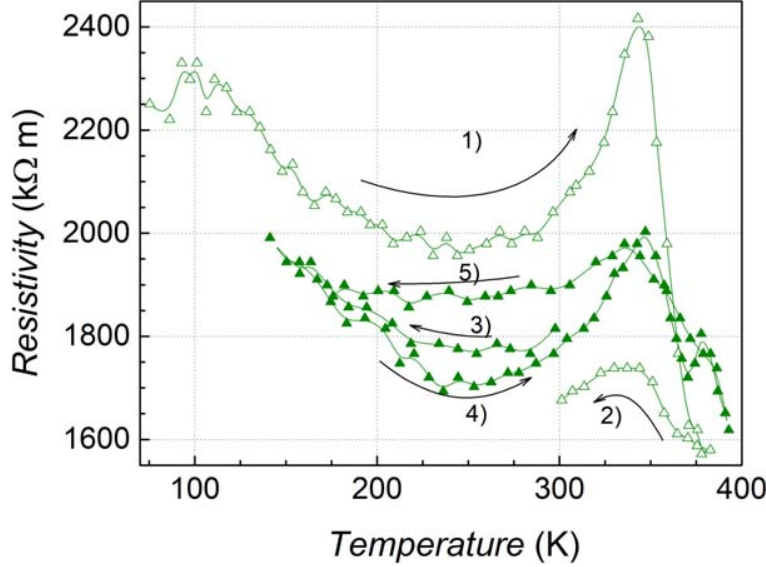

FIG. 4. Dependences of the resistivity for material with 0.08 wt $\%$ MWCNTs during different heating/cooling cycles (at fixed $1 \mathrm{~V}$ voltage; arrows indicate direction of temperature variation). [Color figure can be viewed at wileyonlinelibrary.com]

matrix, thereby leading to the increase of the distance between conductive nanoparticles clusters at the nanoscale and decreasing the electron tunneling current [35]. Thus, MWCNTs network is partially destroyed when the matrix expands. For composites with $0.3 \mathrm{wt} \%$ MWCNTs this effect is less noticeable as the distance between the conductive particles is small and the rapid expansion of the polymer matrix does not destroy the conductive network. At higher temperatures (above $340 \mathrm{~K}$ ) the resistivity decreases with temperature, due to the onset of electrical conductivity in matrix.

Tunneling, transport through defects' centers, and hopping are the most usually observed charge carrier transport mechanisms in composite materials [36]. The dominant mechanism is determined by the type and dimensions of the carbon particles, the distance between them, their orientation and distribution in the matrix [11]. Dependencies of the resistivity on voltage and on temperature have the same character irrespective of CNTs grafting and density of inclusions in the investigated materials. Comparing epoxy-grafted and amino-grafted materials with $0.3 \mathrm{wt} \%$, it is found that the resistivity of epoxygrafted material is larger (the percolation threshold is higher for amino-grafted material [15]) (Figs. 2 and 3). The obtained resistance dependencies for the investigated materials that are above the percolation threshold show that amino-grafted MWCNTs have larger density of charge carrier states at the surface of nanotubes, what increases probability of electron tunneling and leads to larger conductivity of the material.

The resistivity of composite with $0.08 \mathrm{wt} \%$ MWCNTs varies depending on heating/cooling cycle and direction of temperature variation (Fig. 4). This phenomenon was not observed for materials with $0.3 \mathrm{wt} \%$ MWCNTs. The effect can be explained by redistribution of MWCNTs due rapid expansion of polymer matrix [37]. If the concentration of conductive particles in composite is far above the percolation threshold, the distance between 

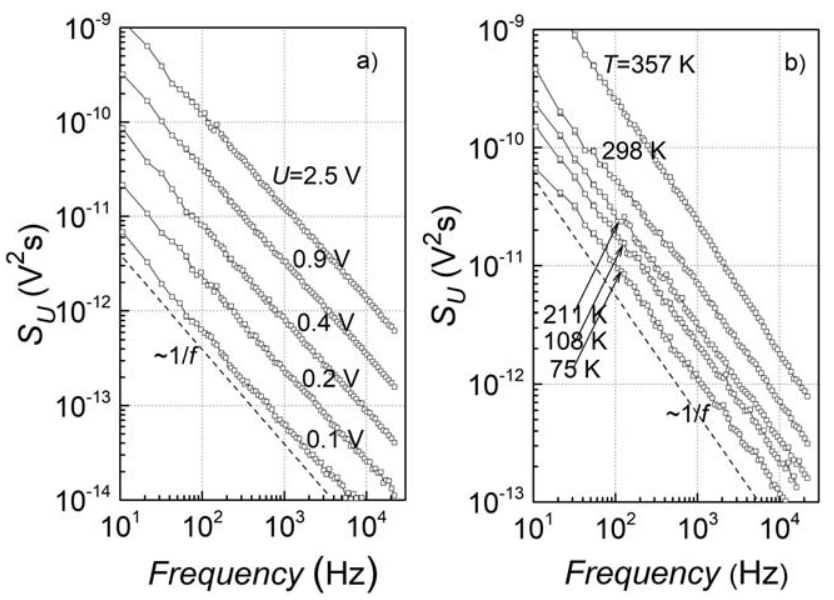

FIG. 5. Spectra of voltage noise for amino-grafted composite with 0.3 wt \% MWCNTs at different voltages at room temperature (a) and at different temperatures at fixed voltage $(U=1.38 \mathrm{~V})(\mathrm{b})$.

particles is very small and the conductivity is less dependent on the orientation and distribution of MWCNTs. When concentration of MWCNTs is close to the percolation threshold $(0.08 \mathrm{wt} \%)$, this redistribution has significant influence on the resistivity of the material.

\section{Noise Properties}

The low-frequency noise spectra of all investigated materials are $1 / f^{\alpha}$-type (where $\alpha$ is close to 1 ) at room temperature (Fig. 5). 1/f -type noise is caused by superposition of many charge carrier capture and release processes with widely distributed relaxation times and similar intensity. At different temperatures noise spectra slightly deviates from $1 / f$ proportionality: the influence of individual Lorentzians (caused by more intensive carrier capture and release processes) is observed (Fig. 5b). As Lorentzian components in noise spectra appear for the composites with both types of MWCNTs, amino-grafted and epoxy-grafted, we may conclude that the individual carrier capture and release centers are not related with a surface of MWCNTs. Carrier capture and release centers are formed by defects in the polymer matrix and are not influenced by the features of CNTs. In the material with high density of conductive particles $(0.3 \mathrm{wt} \%)$ the carrier transport mechanism inside MWCNT is that one which governs noise characteristics. The transport between CNTs is less important for noise characteristics. Just at particular temperature, some defects (that create localized states and influence transport between CNTs) manifest. In material with $0.08 \mathrm{wt} \%$ MWCNTs the carrier transport between CNTs is significant-it is carrier tunneling with assistance of defects' created carrier capture and release centers. State of such center (with captured carrier or without) strongly influences the potential profile in which carriers are moving. The superposition of fluctuations of the potential profile leads to the intensive $1 / f$-type noise.

The spectral density of voltage fluctuation for composites with $0.3 \mathrm{wt} \%$ MWCNTs is approximately
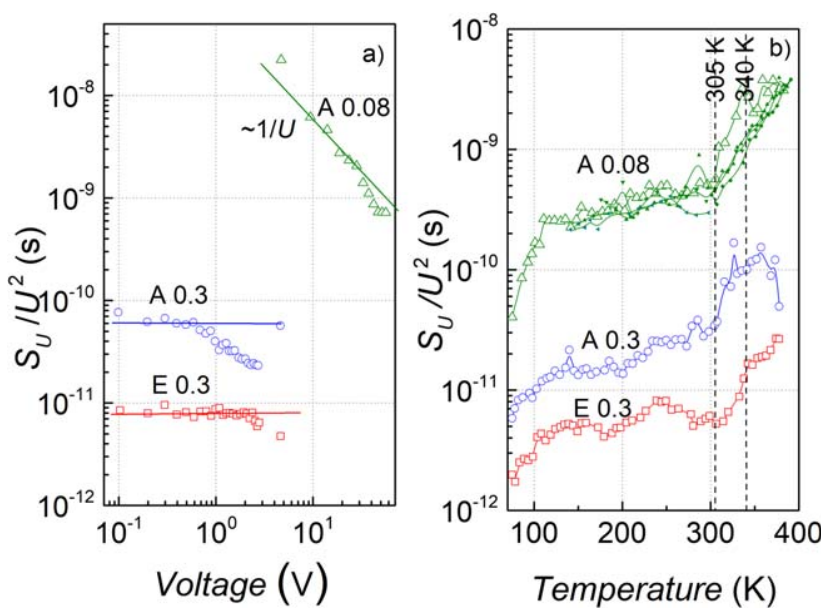

FIG. 6. Normalized voltage noise spectral density dependences on voltage for MWCNT composites at room temperature (a) and voltage noise spectral density dependences on temperature at fixed voltage (b) at 86 $\mathrm{Hz}$ frequency (additional lines with solid symbols in graph (b) by the curve A0.08 represent noise measurements for this sample during different heating/cooling cycles). [Color figure can be viewed at wileyonlinelibrary.com]

proportional to the squared voltage while for the material with $0.08 \mathrm{wt} \%$ inclusions it is directly proportional to the voltage (Fig. 6a). The proportionality of the voltage spectral density to the squared voltage is characteristic for resistance fluctuations. The tunneling between CNTs controlled by the charge carrier capture in localized states (what causes resistance fluctuations) between them determines the conductivity in investigated materials and leads to the observed noise characteristics. Again, this feature is related more to the density of MWCNTs, but not to their surface treatment in the investigated materials.

Nevertheless, some noise differences were observed for amino- and epoxy-grafted composites with the same density of MWCNTs: it is noticed that spectral density of voltage fluctuations for amino-grafted material is about an order of magnitude higher comparing to the epoxygrafted one (Fig. 6). Covering of MWCNTs with additional polyethylene polyamine layer leads to the CNT surface modification: noise measurement results show that amino grafted CNTs distinguish by larger density of surface states, that is, larger number of charge carrier capture centers. The influence of CNTs surface treatment to the noise characteristics is observable in materials with high density of conductive particles, as for material close to the percolation threshold (sample A0.08) influence of CNTs' properties is masked by defects in the matrix.

The variation of noise intensity of investigated materials with temperature is minor at temperatures below $\sim 305 \mathrm{~K}$ (Fig. 5b). However, the decrease of noise intensity below $100 \mathrm{~K}$ is typical for tunneling conductivity [38]. More rapidly the noise spectral density increases with temperature in temperature range from 305 to $340 \mathrm{~K}$, above which the noise increase again goes to saturation. Let us suppose that the resistivity dependence on 
temperature also changes approximately at the same temperature range (Fig. 3): the resistivity stops to decrease at $240-340 \mathrm{~K}$ and rapidly decreases above $340 \mathrm{~K}$. Thus, the more rapid noise intensity increase is observed at the temperature range, where change of conductivity mechanisms occurs, that is, the matrix expansion occurs and then matrix conductivity becomes important. These noise features are observed for all types of investigated composites.

It is important to mention that resistivity dependence on temperature and heating/cooling cycle for material with 0.08 wt $\%$ MWCNTs was not stabile (Fig. 4), but noise characteristics are almost stable during different measuring cycles (Fig. 6b). Conduction of such material is highly influenced by MWCNTs distribution and orientation in matrix what changes with measuring cycle and temperature. Moreover, noise characteristics are more influenced by defects' created carrier capture and release centers, their number and properties.

Type of low-frequency noise and resistivity dependences for both investigated materials: amino- and epoxy-grafted MWCNTs composites, is similar in the investigated voltage and temperature ranges. Some differences are observed only in resistivity values and noise intensity. Thus, different surface of MWCNTs does not change the dominant conduction mechanisms in the investigated composites. As amino-grafted material is known by smaller resistivity and more intensive low-frequency noise, covering of MWCNT's surface by polyethylene polyamine causes the additional surface states and increases the conductivity of overall composite, leading to more intensive low-frequency fluctuations.

\section{CONCLUSIONS}

Conduction mechanisms were investigated in epoxybased MWCNT composites via low-frequency noise characteristics measurement. Two types of MWCNTs with different surface treatment were used for composites fabrication and materials with two MWCNTs density levels: well above and just close to the percolation threshold, were investigated. The dominant conduction mechanisms in the investigated composites with MWCNTs are tunneling inside CNTs and between them controlled by the random charge carrier capture and release processes in localized states in the polymer matrix. The polymer expansion and redistribution of MWCNTs inside the polymer matrix was proved to have a huge impact on the composite close to the percolation threshold resistivity, however less on the low-frequency fluctuations.

MWCNTs' surface treatment with liquid epoxy resin and polyethylene polyamine hardener has no influence on the prevailing conduction mechanisms in the investigated epoxy composites. However, grafting was demonstrated to change the density of surface states at the MWCNT surface: the interface polymer layer formed on the aminografted MWCNT/epoxy composites increases the number of surface charge carrier capture centers and leads to lower resistivity and larger low-frequency fluctuations.

\section{ACKNOWLEDGEMENTS}

Polina Kuzhir is thankful for support by Tomsk State University Competitiveness Improvement Program. Rumiana Kotsilkova is thankful for the support by COST CA15114 AMICI, contract grant 0901/37-2016 of National Science Fund of Bulgaria. Authors are also thankful for support by COST 15107.

\section{REFERENCES}

1. T. Kawaharaa, S. Yamaguchia, Y. Ohnob, K. Maehashib, K. Matsumotob, S. Mizutanic, and K. Itakad, Appl. Surface Sci., 267, 101 (2013).

2. T. Li, X. Liu, C. Dong, and L. Yin, Microelectron. Eng., 119, 155 (2014).

3. A. Maffucci, IEEE Computer Society Annual Symposium on VLSI, 450, (2015). (2015).

4. Y.S. Mehrabani, and M. Eshghi, IEEE Transactions on Very Large Scale Integration (VLSI) Systems, vol. 24, 3268 (2016).

5. S. Agarwal, S K Manhas, S. Dasgupta and N. Jain, 2016 29th International Conference on VLSI Design and 2016 15th International Conference on Embedded Systems, Vol. 361 (2016).

6. B.D. Vivo, P. Lamberti, G. Spinelli, and V. Tucci, J. Appl. Phys., 115, 154311 (2011).

7. B.D. Vivo, P. Lamberti, G. Spinelli, and V. Tucci, J. Appl. Phys., 115, 54311 (2014).

8. Q. Cao, J. Tersoff, D.B. Farmer, Y. Zhu, and S.-J. Han, Science, 356, 1369 (2017).

9. C. Qiu, Z. Zhang, M. Xiao, Y. Yang, D. Zhong, and L.-M. Peng, Science, 355, 271 (2017).

10. C.-S. Lee, E. Pop, and H.-S. P. Wong, Compact Modeling and Design Optimization of Carbon Nanotube Field-Effect Transistors for the Sub-10-nm Technology Nodes. IEEE Device Research Conference, vol. 275 (2015).

11. A.K. Ghavidel, T. Azdast, M. Shabgard, A. Navidfar, and S. Sadighikia, J. Appl. Polym. Sci., 132, n/a (2015).

12. J. Macutkevic, P. Kuzhir, A. Paddubskaya, M. Shuba, J. Banys, S. Maksimenko, V.L. Kuznetsov, I.N. Mazov, and D.V. Krasnikov, Phys. Stat. Sol (A), 210, 2491 (2013).

13. T.W. Ebbesen, H.J. Lezec, H. Hiura, J.W. Bennett, H.F. Ghaemi, and T. Thio, Nature, 382, 54 (1996).

14. S. Cogal, F. Bozduman, G. Yurdabak and A. Oksuz, Modification of carbon nanotube withpoly(3,4-ethylenedioxythiophene) byusing rf rotating plasma. 2015 42nd IEEE International Conference on Plasma Sciences (ICOPS) (2016).

15. R. Kotsilkova, E. Ivanov, D. Bychanok, A. Paddubskaya, M. Demidenko, J. Macutkevic, S. Maksimenko, and P. Kuzhir, Compos. Sci. Technol., 106, 85 (2015).

16. F.L. Jin, and S.J. Park, Carbon Lett., 14, 1 (2013).

17. G. Sassine, F. Martinez, F. Pascal, and A. Hoffmann, IEEE Proceedings of the Noise and Fluctuations (ICNF), Vol. 475, (2011).

18. L. Guadagno, M. Raimondo, V. Vittoria, L. Vertuccio, K. Lafdi, B. De Vivo, P. Lamberti, G. Spinelli, and V. Tucci, Nanotechnology, 24, 305704 (2013). 
19. C. Barone, F. Romeo, and S. Pagano, IEEE Proceedings of the Noise and Fluctuations (ICNF), Vol. 1, (2013).

20. S. Farhana, A.H.M.Z. Alam, S. Khan, and S.M.A. Motakabber, The 5th IEEE National Symposium on Information Technology: Towards Smart World, Vol. 1, (2015).

21. V. Palenskis, J. Matukas, J. Vyšniauskas, S. Pralgauskaite, H. Shtrikman, D. Seliuta, I. Kašalynas, and G. Valušis, Fluct. Noise Lett., 12, 1350014 (2013).

22. V. Palenskis, J. Matukas, S. Pralgauskaite, D. Seliuta, I. Kašalynas, L. Subačius, G. Valušis, S.P. Khanna, and E.H. Linfield, J. Appl. Phys., 113, 083707 (2013).

23. S. Pralgauskaite, V. Palenskis, J. Matukas, B. Šaulys, V. Kornijčuk, and V. Verdingovas, Solide-State Electron., 79, 104 (2013).

24. B.K. Jones, IEEE Trans. Electron. Dev., 41, 2188 (1994).

25. E.S. Snow, J.P. Novak, M.D. Lay, and F.K. Perkins, Appl. Phys. Lett., 85, 4172 (2004).

26. P.G. Collins, M.S. Fuhrer, and A. Zettl, Appl. Phys. Lett., 76, 894 (2000).

27. C. Barone, S. Pagano, and H.C. Neitzert, Appl. Phys. Lett., 97, 152107 (2010).

28. J. Macutkevic, P.P. Kuzhir, A.G. Paddubskaya, J. Banys, S.A. Maksimenko, E. Stefanutti, F. Micciulla, and S. Bellucci, J. Nanophotonics, 7, 073593 (2013).
29. J. Banys, T. Ramoška, J. Matukas, S. Pralgauskaite, F.M.M. Alawneh, V.V. Shvartsman, and D.C. Lupascu, Ferroelectrics, 417, 25 (2011).

30. P. Sheng, Phys. Rev. B, 21, 2180 (1980).

31. T. Okamoto, M. Koyama, Y. Inoue, T. Tomimura, S. Nakamura, 2001 IEEE 7th International Conference on Solid Dielectrics, Vol. 117 (2001).

32. A. Mdahari, F. Carmona, C. Brosseau, and P. Delhaes, J. Appl. Phys., 103, 054303 (2008).

33. P. Sheng, E.K. Sichel, and J.I. Gittleman, Phys. Rev. Lett., 40, 1197 (1978).

34. J. Zhou, and J.P. Lucas, Polymer, 40, 5513 (1999).

35. J. Macutkevic, P. Kuzhir, A. Paddubskaya, S. Maksimenko, J. Banys, A. Celzard, V. Fierro, S. Bistarelli, A. Cataldo, F. Micciulla, and S. Bellucci, J. Appl. Phys., 114, 033707 (2013).

36. D. van der Putten, S.T. Moonen, H.B. Brom, J.C.M. Brookken-Zijp, and M.A.J. Michels, Phys. Rev. Lett., 69, 494 (1992).

37. T.A. Ezquerra, M.T. Connor, S. Roy, M. Kulescza, J. Fernandes-Nascimento, and F.J. Baltá-Calleja, Compos. Sci. Technol., 61, 903 (2001).

38. S. Kogan, Electronic Noise and Fluctuations in Solids. Cambridge University Press, Cambridge (1996). 